\title{
Superantigen gene profile, emm type and antibiotic resistance genes among group A streptococcal isolates from Barcelona, Spain
}

\begin{abstract}
Correspondence
Ferran Navarro

fnavarror@santpau.es
\end{abstract}

Received 21 December 2005

Accepted 18 April 2006

\author{
Alba Rivera, ${ }^{1,4}$ Montserrat Rebollo, ${ }^{1}$ Elisenda Miró, ${ }^{1}$ Míriam Mateo, ${ }^{2}$ \\ Ferran Navarro, ${ }^{1,4}$ Mercé Gurguí, ${ }^{3}$ Beatriz Mirelis ${ }^{1,4}$ and Pere Coll ${ }^{1,4}$ \\ ${ }^{1,2,3}$ Servei de Microbiologia ${ }^{1}$, Servei de Medicina Interna ${ }^{2}$ and Unitat de Malalties Infeccioses ${ }^{3}$, \\ Hospital de la Santa Creu i Sant Pau, Avda Sant Antoni M ${ }^{a}$ Claret 167, 08025 Barcelona, \\ Spain \\ ${ }^{4}$ Unitat de Microbiologia, Departament de Genètica i Microbiologia, Universitat Autònoma de \\ Barcelona, 08193 Cerdanyola del Vallès (Bellaterra), Spain
}

\begin{abstract}
Group A streptococcus (GAS) has been described as an emerging cause of severe invasive infections. A retrospective hospital-based study was conducted, including GAS isolates causing invasive or non-invasive infections from January 1999 to June 2003 in Barcelona. Demographic and clinical information on the invasive cases was obtained from medical files. GAS isolates collected from 27 patients with invasive infections and 99 patients with non-invasive infections were characterized by emm type and subtype, superantigen (SAg) gene profile (speA-C, speF-J, speL, speM, ssa and smeZ), allelic variants of $s p e A$ and smeZ genes, antibiotic susceptibility and genetic resistance determinants. The most prevalent emm type was emm1 (17.5\%), followed by emm3 $(8 \cdot 7 \%)$, emm4 (8.7\%), emm $12(7 \cdot 1 \%)$ and emm28 (7·1\%). The smeZ allele and SAg gene profiles were closely associated with the emm type. The speA2, speA3 and speA4 alleles were found in emm 1, emm3 and emm6 isolates, respectively. Overall, 27.8, 25.4 and $11.9 \%$ of isolates were resistant to erythromycin, tetracycline or both agents, respectively. Reduced susceptibility to ciprofloxacin and levofloxacin (MIC 2-4 $\mu \mathrm{g} \mathrm{ml}^{-1}$ ) was found in $3.2 \%$ of isolates. mef(A)-positive emm types 4, 12 and 75, and erm(B)-positive emm types 11 and 25 were responsible for up to $80 \%$ of the erythromycin-resistant isolates. No significant differences in emm-type distribution, SAg gene profile or resistance rates were found between invasive and non-invasive isolates. The SAg and antibiotic resistance genes appeared to be associated with the emm type and were independent of the disease type.
\end{abstract}

\section{INTRODUCTION}

Group A streptococcus (GAS) is a human pathogen that is responsible for a wide array of infections, varying in severity from acute pharyngitis and impetigo to severe invasive infections such as necrotizing fasciitis (NF) and streptococcal toxic shock syndrome (STSS) (Cunningham, 2000).

The resurgence and persistence of severe forms of GAS diseases reported since the mid 1980s (Hoge et al., 1993; Stevens, 2002) has motivated intensive research on epidemiological, microbiological and clinical aspects of invasive GAS disease. Several factors have been considered to explain

Abbreviations: GAS, group A streptococcus; NF, necrotizing fasciitis; SAg, superantigen; STSS, streptococcal toxic shock syndrome.

The GenBank/EMBL/DDBJ accession numbers for emm1.25, emm12.27, emm83.4, smeZ-35, smeZ-36, smeZ-37, smeZ-38 and smeZ-39 are AY686603, AY742805, AY742806, AY965265, AY965878, AY965879, D0001538 and D0001539, respectively. differences in disease frequency and severity, including changes in the virulence of the bacterium (Banks et al., 2002; Musser et al., 1993) and the role of host immunity (Åkesson et al., 2004; Basma et al., 1999; Kotb et al., 2002; NorrbyTeglund et al., 2000).

Among the many factors involved in the virulence of the pathogen, the $\mathrm{M}$ protein and a group of exotoxins known as streptococcal superantigens (SAgs) have received considerable attention. Sequence analysis of the emm gene (encoding the $\mathrm{M}$ protein) has become an important surveillance tool for investigating the dynamics of GAS infection and more than $150 \mathrm{emm}$ gene sequence types and several $\mathrm{emm}$ subtypes have been documented (Facklam et al., 2002; Li et al., 2003).

SAgs are thought to contribute to the pathogenesis of severe GAS infections by virtue of their potent immunostimulatory activity (Norrby-Teglund et al., 2001). The gene distribution 
of SAgs has been used as an additional epidemiological tool to explore genomic heterogeneity and the possible correlation between toxin gene content and disease type (Chatellier et al., 2000; Schmitz et al., 2003; Vlaminckx et al., 2003).

To improve our understanding of severe GAS disease in Europe, the Strep-EURO project was recently set up (Lamagni et al., 2005; http://www.strep-euro.lu.se). To our knowledge, no studies on GAS epidemiology and SAg distribution, including invasive and non-invasive GAS isolates, have been conducted to date in Spain. Surveillance that includes invasive and non-invasive GAS isolates is important to evaluate epidemiological changes in GAS diseases and to distinguish between virulence properties and the prevalence of a particular GAS strain in the general population (Johnson et al., 2002; O'Brien et al., 2002).

In this study, we report clinical and epidemiological data on invasive and non-invasive GAS infections from a tertiary care hospital in Barcelona. emm subtype distribution, SAg gene profiles (speA-C, speF-J, speL, speM, ssa and smeZ) and allelic variants of speA and smeZ genes were used to evaluate possible differences between GAS isolates causing invasive or non-invasive infections. The prevalence and mechanisms of macrolide, tetracycline and fluoroquinolone resistance were also determined.

\section{METHODS}

Bacterial isolates and patients. This retrospective study included GAS clinical isolates collected from January 1999 to June 2003 at Hospital de la Santa Creu i Sant Pau (Barcelona, Spain), a 645-bed tertiary care hospital covering a population of 300000 . Only one isolate per patient was included. Clinical sample procedures and bacterial identification were performed according to standard methods (Ruoff et al., 2003). All isolates were stored at $-80{ }^{\circ} \mathrm{C}$ in trypticase soy broth supplemented with $10 \%$ glycerol until testing.

Invasive infection was defined as the recovery of GAS from sites that are usually sterile, including blood, cerebrospinal, peritoneal, pleural and joint fluids, deep tissue abscesses and a superficial site in clinical association with STSS or NF. STSS was defined according to the consensus definition of the Working Group on Severe Streptococcal Infections (1993). Non-invasive isolates were recovered from sites that are usually non-sterile (skin, wound, ear, genital, throat and other miscellaneous sites) and were included for genetic comparison with invasive isolates. Demographic and clinical data on patient characteristics, course of infection and outcome were recorded from the medical history. Medical records were also examined to assess underlying diseases or conditions that may have predisposed patients to invasive disease, such as skin trauma, injection drug use, varicella infection, alcohol abuse or decreased body defences (malignancy, HIV infection, diabetes and immunosuppressive therapy). The overall fatality rate was assessed 30 days after the date of specimen sampling.

Antimicrobial susceptibility testing. The susceptibility to penicillin $\mathrm{G}$, erythromycin, clindamycin, tetracycline and levofloxacin was determined by using the disc-diffusion method, according to Clinical and Laboratory Standards Institute guidelines (National Committee for Clinical Laboratory Standards, 2003) using commercial discs (Bio-Rad). Macrolide resistance phenotypes [constitutive (cMLSb), inducible (iMLSb) and M phenotype] were determined by using the double-disc test, as described by Seppälä et al. (1993). MICs of penicillin G, cefotaxime and levofloxacin were determined for all isolates by using Etest (ABBiodisk), according to the manufacturer's recommendations.

emm typing. The emm gene was amplified and sequenced as described previously (Rivera et al., 2005) and by Beall et al. (1996). emm type and subtype assignments were determined as described on the Centers for Disease Control and Prevention (CDC) website (http://www.cdc.gov/ncidod/biotech/strep/doc.htm).

Detection of toxin genes. GAS isolates were tested for the presence of speA, speC, speH, speI, speJ, speL, speM, ssa and smeZ by PCR, using the primers listed in Table 1. With the exception of speL, speM and smeZ, two different primer pairs were used. PCRs were performed using the following conditions: denaturing for $5 \mathrm{~min}$ at $94{ }^{\circ} \mathrm{C}, 30$ cycles for $30 \mathrm{~s}$ at $94^{\circ} \mathrm{C}$, annealing for $30 \mathrm{~s}$ at the temperature determined for each primer pair (Table 1) and elongation for $1 \mathrm{~min}$ at $72{ }^{\circ} \mathrm{C}$, followed by a final elongation step at $72{ }^{\circ} \mathrm{C}$ for $10 \mathrm{~min}$.

Sequences of all speA genes were determined and aligned with published sequences in GenBank. Similarly, the sequences of smeZ genes of 95 selected isolates representing all $\mathrm{emm}$ types and subtypes, including those isolates with different toxin profiles, were also determined.

The presence of $s p e B, s p e F$ and $s p e G$ was determined for all isolates by slot-blot analysis (Schleicher \& Schuell). DNA samples were obtained as described by Pan \& Fisher (1996). DNA probes used in hybridization assays were obtained by PCR with the same conditions as described above using the primers for $s p e B, s p e F$ and $s p e G$ given in Table 1 . Labelling of probes and hybridization and detection were performed using ECL Direct Nucleic Acid Labelling (Amersham Biosciences). Hybridization results were confirmed by PCR in 38 randomly selected isolates. Previously published GAS strains, kindly provided by $\mathrm{Dr}$ T. Proft, were used as positive controls for the detection of toxin genes, including FP 4223 (positive for speG, speJ, speM and smeZ), FP 5417 (positive for speA, speG, speJ, speL and smeZ), FP 5971 (positive for speA, speC, speG, speH, speJ and smeZ), 1/5045 (positive for speA, speC, speG, speJ, ssa and smeZ) and 85/167 (positive for speG, speH, speI, speJ and smeZ) (Proft et al., 2003).

Identification of resistance genes. Resistance determinants $\operatorname{erm}(\mathrm{A})$ subclass erm(TR) [hereafter designated $\operatorname{erm}(\mathrm{TR})$ ], erm(B) and $m e f(\mathrm{~A})$ were detected in erythromycin-resistant isolates by PCR with the primers listed in Table 1, using the same conditions as described for the toxin genes, except for mef(A) (denaturing for $5 \mathrm{~min}$ at $94{ }^{\circ} \mathrm{C}, 30$ cycles for $1 \mathrm{~min}$ at $94^{\circ} \mathrm{C}$, annealing for $1 \mathrm{~min}$ at $60^{\circ} \mathrm{C}$ and elongation for $1 \mathrm{~min} 30 \mathrm{~s}$ at $72^{\circ} \mathrm{C}$, followed by $10 \mathrm{~min}$ at $\left.72^{\circ} \mathrm{C}\right)$. In tetracycline-resistant isolates, $\operatorname{tet}(\mathrm{M})$ and $\operatorname{tet}(\mathrm{O})$ were also detected by PCR using primers described by $\mathrm{Ng}$ et al. (2001). Characterization of mutational changes in parC, parE, gyrA and gyrB were determined previously in isolates with a levofloxacin MIC of $\geqslant 2 \mu \mathrm{g} \mathrm{ml}^{-1}$ (Rivera et al., 2005).

Statistical analysis. Chi square or Fisher's exact test was used for statistical analysis. A $P$ value of $<0.05$ was considered significant. The SPSS 11.5 statistical package was used for all analyses.

\section{RESULTS AND DISCUSSION}

\section{Clinical findings}

A total of 126 isolates were recovered, $27(21 \cdot 4 \%)$ from patients with invasive infections and $99(78.6 \%)$ from noninvasive cases. Skin and soft tissue infections, in the form of cellulitis (five cases), NF (four cases), thrombophlebitis (one case) and bacterial infection secondary to varicella (one 
Table 1. Primers used for amplification of $s p e A, s p e B, s p e C, s p e F, s p e G$, speH, spel, speJ, speL, speM, ssa and smeZ genes and for sequencing of speA and smeZ genes

\begin{tabular}{|c|c|c|c|c|c|}
\hline Gene & Forward primer $\left(5^{\prime}-3^{\prime}\right)$ & Reverse primer $\left(5^{\prime}-3^{\prime}\right)$ & $\begin{array}{l}\text { Amplicon } \\
\text { size (bp) }\end{array}$ & $\begin{array}{c}\text { Annealing } \\
\text { temperature }\left({ }^{\circ} \mathrm{C}\right)\end{array}$ & Reference \\
\hline speA & ACT(T/C)AAGAACC(A/G)AGAGATG $(\mathrm{G} / \mathrm{T})^{*}$ & CTTTAT(T/C)CTTAG(G/A)TATGAAC* & 356 & 50 & Modified. Chatellier et al. (2000) \\
\hline speA & CCAAGCCAACTTCACAGATC & CTTTAT(T/C)CTTAG(G/A)TATGAAC* & 523 & 50 & This study \\
\hline speB & CAACCAGTTGTTAAATCTCT & CTAAGGTTTGATGCCTACAA & 762 & $58 \cdot 3$ & Modified. Chatellier et al. (2000) \\
\hline speC & AAGTGACTCTAAGAAAGACA & TTGAGTATCAATGTTTAATG & 130 & 50 & Chatellier et al. (2000) \\
\hline speC & TGTCTTATGAGGCCTCTC & ATCTGATCTAGTCCCTTC & 386 & 50 & This study \\
\hline speF & CGAAATTAGAAAAGAGGAC & GGCTGAGCAAAAGTGTGTG & 1193 & $57 \cdot 3$ & This study \\
\hline speG & GATGAAAATTTAAAAGATTTAA & GGGGGGAGAATAGCACTAGT & 648 & 50 & Modified. Chatellier et al. (2000) \\
\hline speH & AATTCTTATAATACAACCAA & TTAGCTGATTGACACATCTA & 615 & 52 & Modified. Chatellier et al. (2000) \\
\hline speH & CACATATTGATAAGAAAATCTACAGC & GAAATTGAGTTGAGTCTATTCTCTCG & 666 & 59 & Igwe et al. (2003) \\
\hline speI & ATGAGTAGTGTGGGAGTTATTAA & TTATTTATTAAATTTAACTAAG & 678 & 50 & Proft et al. (2001) \\
\hline speI & CTTTGGAGTATTCTCСТCСС & СТСТСТСТGTCACCATGTCC & 382 & 59 & This study \\
\hline speJ & GATAGTGAAAATATTAAAGACG & GCTCCTATCTTATTTAGTCC & 639 & 55 & Modified. Proft et al. (2001) \\
\hline speJ & GATAGTGAAAATATTAAAGACG & TTATTTAGTCCAAAGGTAAATATC & 630 & 55 & Modified. McCormick et al. (2001) \\
\hline speJ & GTTATAATAATCTTTCATGGGTACGG & CTTTCATGTTTATTGCCATTGATCGC & 545 & 59 & Igwe et al. (2003) \\
\hline speL & TTAGGATGGTTTCTGCGGAAGAGAC & TTCCTCTTTCTCGCCTGAGCCGTG & 596 & 60 & Igwe et al. (2003) \\
\hline speM & GCTCTATACACTACTGAGAGTGTC & CATATCAATCGTTTCATTATCTG & 612 & 56 & Igwe et al. (2003) \\
\hline ssa & AGTCAGCCTGACCCTACTCC & TAAGGTGAACCTCTATAGCT & 691 & 57 & Modified. Chatellier et al. (2000) \\
\hline$s s a$ & GTGCACAATTATTATCGATTAGTG & GGTGAACCTCTATAGCTATAGCTGAAG & 723 & 60 & Igwe et al. (2003) \\
\hline smeZ & TAGAAGTAGATAATAATTCC $\dagger$ & TTAGGAGTCAATTTCTATAT $\dagger$ & 629 & $48 \cdot 3$ & Modified. Chatellier et al. (2000) \\
\hline $\operatorname{erm}(\mathrm{TR})$ & TTATAACCGGCAAGGAGAAG & GCTTCAGCACCTGTCTTAATTGAT & 678 & 60 & Modified. Reinert et al. (2003) \\
\hline $\operatorname{erm}(\mathrm{B})$ & CGAGTGAAAAAGTACTCAACC & GGCGTGTTTCATTGCTTGATG & 617 & 60 & Weber et al. (2001) \\
\hline$m e f(\mathrm{~A})$ & TATGACAGCCTCAATGCGT & ACCGATTCTATCAGCAAAGA & 1435 & 60 & Modified. Reinert et al. (2003) \\
\hline
\end{tabular}

*Primers used for sequencing the speA gene.

†Primers used for sequencing the smeZ gene. 
Table 2. Distribution of emm types according to type of infection, toxin gene profile and macrolide and tetracycline resistance genes

Abbreviations: Ery, erythromycin; Tet, tetracycline. +, Present; -, absent.

\begin{tabular}{|c|c|c|c|c|c|c|c|c|c|c|c|c|c|c|}
\hline \multirow{2}{*}{$\begin{array}{l}\text { emm subtype } \\
\text { (no. of strains) }\end{array}$} & \multirow{2}{*}{$\begin{array}{c}\text { No. invasive/ } \\
\text { non-invasive } \\
\text { cases }\end{array}$} & \multicolumn{10}{|c|}{ Toxin gene profile } & \multicolumn{2}{|c|}{ No. of strains resistant to: } & \multirow{2}{*}{$\begin{array}{l}\text { Resistance gene } \\
\text { (no. of strains) }\end{array}$} \\
\hline & & $\begin{array}{r}\text { speA } \\
\text { allele }\end{array}$ & speC & speG & speH & speI & speJ & speL & speM & $s s a$ & $\begin{array}{l}\quad s m e Z \text { allele } \\
\text { (no. positive/no. } \\
\text { tested strains) }\end{array}$ & Ery & Tet & \\
\hline emm1.0 (11) & $0 / 11$ & speA2 & - & + & - & - & + & - & - & - & $(6 / 6)^{*}$ & 1 & 0 & $\operatorname{erm}(\mathrm{B})(1)$ \\
\hline emm1.25 (11) & $2 / 9$ & speA2 & - & + & - & - & + & - & - & - & $(6 / 6)^{*}$ & 0 & 0 & - \\
\hline emm2.0 (2) & $1 / 1$ & - & + & + & - & - & - & + & + & - & $(0 / 2)$ & 0 & 0 & - \\
\hline emm2.0 (1) & $0 / 1$ & - & + & + & - & - & - & - & + & - & $(0 / 1)$ & 0 & 0 & - \\
\hline emm2.0 (1) & $0 / 1$ & - & - & + & - & - & - & - & + & - & $(0 / 1)$ & 0 & 0 & - \\
\hline emm3.1 (10) & $4 / 6$ & speA3 & - & + & - & - & - & - & + & + & $(6 / 6)^{*}$ & 0 & 0 & - \\
\hline emm3.2 (1) & $0 / 1$ & speA3 & - & + & - & - & - & - & + & + & $(1 / 1)^{*}$ & 0 & 0 & - \\
\hline emm4.0 (10) & $3 / 7$ & - & + & - & - & - & - & - & - & + & smeZ-8 (5/5) & 9 & 0 & $m e f(\mathrm{~A})(9)$ \\
\hline emm4.2 (1) & $0 / 1$ & speA1 & - & - & - & - & - & - & + & - & smeZ-39 (1/1) & 0 & 0 & - \\
\hline emm6.0 (3) & $2 / 1$ & speA4 & + & + & - & - & - & - & + & - & $(3 / 3)^{*}$ & 0 & 0 & - \\
\hline emm9.0 (4) & $2 / 2$ & - & - & + & - & - & - & - & - & - & smeZ-36 (4/4) & 0 & 0 & - \\
\hline emm11.0 (7) & $1 / 6$ & - & + & + & + & + & - & - & - & - & smeZ-30/31 (4/4) & $7 \dagger$ & 7 & $\operatorname{erm}(\mathrm{B})(7)$ \\
\hline emm11.0 (1) & $0 / 1$ & - & + & + & - & - & - & - & - & - & smeZ-30/31 (1/1) & $1 \dagger$ & 1 & $\operatorname{erm}(\mathrm{B})+\operatorname{tet}(\mathrm{M})(1)$ \\
\hline emm12.0 (3) & $0 / 3$ & - & - & + & + & + & - & - & - & - & smeZ-3 (3/3) & 0 & 0 & - \\
\hline emm12.0 (1) & $1 / 0$ & - & + & + & + & + & - & - & - & - & smeZ-3 (1/1) & 0 & 0 & - \\
\hline emm12.0 (1) & $0 / 1$ & - & - & + & + & + & - & - & - & + & smeZ-3 (1/1) & 1 & 0 & $m e f(\mathrm{~A})(1)$ \\
\hline emm12.1 (1) & $0 / 1$ & - & - & + & + & + & - & - & - & - & smeZ-3 (1/1) & 1 & 0 & $m e f(\mathrm{~A})(1)$ \\
\hline emm12.7 (1) & $0 / 1$ & - & - & + & + & + & - & - & - & - & smeZ-3 (1/1) & 1 & 0 & $m e f(\mathrm{~A})(1)$ \\
\hline emm12.18 (1) & $0 / 1$ & - & - & + & + & + & - & - & - & - & smeZ-3 (1/1) & 0 & 0 & - \\
\hline emm12.27 (1) & $0 / 1$ & - & - & + & + & + & - & - & - & - & smeZ-3 (1/1) & 1 & 0 & $m e f(\mathrm{~A})(1)$ \\
\hline emm18.9 (1) & $1 / 0$ & speA1 & + & + & - & - & + & + & + & - & smeZ-13 (1/1) & 0 & 0 & - \\
\hline emm22.0 (1) & $0 / 1$ & - & + & + & - & - & - & - & - & + & smeZ-22 (1/1) & $1 \dagger$ & 1 & $\operatorname{erm}(\mathrm{B})+\operatorname{tet}(\mathrm{M})(1)$ \\
\hline emm22.0 (1) & $1 / 0$ & - & - & + & - & - & - & + & + & + & smeZ-22(1/1) & 0 & 1 & $\operatorname{tet}(\mathrm{M})$ \\
\hline emm25.2 (3) & $2 / 1$ & - & - & + & + & + & - & - & - & + & smeZ-37 (2/2) & $3 \dagger$ & 3 & $\operatorname{erm}(\mathrm{B})+\operatorname{tet}(\mathrm{M})(3)$ \\
\hline emm25.2 (1) & $1 / 0$ & - & + & + & + & + & - & - & - & + & smeZ-37 (1/1) & $1 \dagger$ & 1 & $\operatorname{erm}(\mathrm{B})+\operatorname{tet}(\mathrm{M})(1)$ \\
\hline emm28.0 (6) & $0 / 6$ & - & + & + & - & - & + & - & + & - & smeZ-16 (2/2) & 2 & 0 & $\operatorname{erm}(\mathrm{B})(2)$ \\
\hline emm28.0 (1) & $0 / 1$ & - & + & + & - & - & + & + & + & - & smeZ-16 (1/1) & 0 & 0 & - \\
\hline emm28.0 (1) & $0 / 1$ & speA2 & - & + & - & - & + & - & + & - & smeZ-16 (1/1) & 0 & 0 & - \\
\hline emm28.0 (1) & $1 / 0$ & - & + & + & - & - & + & - & - & - & smeZ-16 (1/1) & 0 & 0 & - \\
\hline emm29.2 (1) & $0 / 1$ & - & - & + & - & - & - & + & + & - & smeZ-17 (1/1) & 0 & 0 & - \\
\hline emm43.5 (1) & $0 / 1$ & - & + & + & - & - & + & - & - & - & smeZ-7 (1/1) & 0 & 1 & $\operatorname{tet}(\mathrm{M})(1)$ \\
\hline emm44/61.0 (1) & $0 / 1$ & - & - & + & + & - & + & - & + & + & $(0 / 1)$ & 0 & 1 & $\operatorname{tet}(\mathrm{M})$ \\
\hline emm44/61.0 (1) & $0 / 1$ & - & - & + & - & - & + & - & - & + & smeZ-4 (1/1) & 0 & 0 & - \\
\hline emm49.3 (1) & $0 / 1$ & speA1 & - & + & + & + & - & - & - & - & smeZ-3 (1/1) & 0 & 1 & $\operatorname{tet}(\mathrm{M})(1)$ \\
\hline
\end{tabular}


Table 2. cont.

\begin{tabular}{|c|c|c|c|c|c|c|c|c|c|c|c|c|c|c|}
\hline \multirow{2}{*}{$\begin{array}{l}\text { emm subtype } \\
\text { (no. of strains) }\end{array}$} & \multirow{2}{*}{$\begin{array}{l}\text { No. invasive/ } \\
\text { non-invasive } \\
\text { cases }\end{array}$} & \multicolumn{10}{|c|}{ Toxin gene profile } & \multicolumn{2}{|c|}{ No. of strains resistant to: } & \multirow{2}{*}{$\begin{array}{l}\text { Resistance gene } \\
\text { (no. of strains) }\end{array}$} \\
\hline & & $\begin{array}{r}\text { speA } \\
\text { allele }\end{array}$ & speC & speg & speH & speI & speJ & speL & speM & ssa & $\begin{array}{l}\quad \text { smeZ allele } \\
\text { (no. positive/no. } \\
\text { tested strains) }\end{array}$ & Ery & Tet & \\
\hline emm50.0 (2) & $1 / 1$ & - & + & + & - & - & - & - & - & - & smeZ-33 (1/1) & 0 & 0 & - \\
\hline emm50.0 (1) & $0 / 1$ & - & - & + & - & - & - & - & + & + & smeZ-35 (1/1) & 0 & 1 & $\operatorname{tet}(\mathrm{M})(1)$ \\
\hline emm58.0 (1) & $0 / 1$ & - & - & + & - & - & - & - & - & + & $s m e Z-28(1 / 1)$ & $1 \dagger$ & 1 & $\operatorname{erm}(\mathrm{TR})+\operatorname{tet}(\mathrm{M})(1)$ \\
\hline emm58.0 (1) & $0 / 1$ & - & + & + & + & - & - & - & - & - & smeZ-28 (1/1) & 0 & 0 & - \\
\hline emm59.0 (2) & $0 / 2$ & - & - & + & - & - & + & - & + & - & smeZ-12 (2/2) & 0 & 2 & $\operatorname{tet}(\mathrm{M})$ \\
\hline emm63.0 (1) & $0 / 1$ & - & - & - & - & - & - & - & - & - & $(0 / 1)$ & 0 & 1 & $\operatorname{tet}(\mathrm{M})(1)$ \\
\hline emm64.0 (2) & $0 / 2$ & - & - & + & - & - & - & - & + & - & smeZ-4 (1/2) & 0 & 0 & - \\
\hline emm70.0 (1) & $0 / 1$ & - & - & + & - & - & - & + & + & - & smeZ-17 (1/1) & 0 & 1 & $\operatorname{tet}(\mathrm{M})(1)$ \\
\hline emm75.0 (2) & $0 / 2$ & - & + & + & + & + & + & + & + & - & smeZ-29 (2/2) & 2 & 0 & $m e f(\mathrm{~A})(2)$ \\
\hline emm75.0 (1) & $0 / 1$ & - & + & + & + & + & - & + & + & - & smeZ-29 (1/1) & 1 & 0 & $m e f(\mathrm{~A})(1)$ \\
\hline emm77.0 (5) & $1 / 4$ & - & + & - & - & - & - & - & - & - & smeZ-37 (5/5) & $1 \dagger$ & 5 & $\begin{array}{l}\operatorname{erm}(\mathrm{TR})+\operatorname{tet}(\mathrm{O})(1) \\
\operatorname{tet}(\mathrm{M})+\operatorname{tet}(\mathrm{O}) \\
\operatorname{tet}(\mathrm{O})(3)\end{array}$ \\
\hline emm77.0 (1) & $1 / 0$ & - & + & + & - & - & - & - & - & - & smeZ-37 (1/1) & 0 & 1 & $\operatorname{tet}(\mathrm{O})$ \\
\hline emm77.0 (1) & $0 / 1$ & - & + & - & + & - & - & - & - & - & smeZ-37 (1/1) & 0 & 0 & - \\
\hline emm77.0 (1) & $1 / 0$ & - & + & - & + & - & - & - & - & + & smeZ-37 (1/1) & 0 & 1 & $\operatorname{tet}(\mathrm{O})$ \\
\hline emm81.0 (1) & $0 / 1$ & - & - & + & + & - & - & - & - & - & $(0 / 1)$ & 1 & 0 & $m e f(\mathrm{~A})(1)$ \\
\hline emm83.4 (1) & $1 / 0$ & - & + & + & - & - & - & + & + & - & smeZ-15 (1/1) & 0 & 1 & $\operatorname{tet}(\mathrm{M})$ \\
\hline emm87.0 (3) & $0 / 3$ & - & + & + & - & - & + & - & - & + & smeZ-5 (2/2) & 0 & 0 & - \\
\hline emm89.0 (5) & $0 / 5$ & - & - & + & - & - & - & - & - & - & smeZ-13 (4/4) & 0 & 0 & - \\
\hline st11014 (1) & $0 / 1$ & - & + & - & + & - & - & - & - & - & smeZ-38 (1/1) & 0 & 1 & $\operatorname{tet}(\mathrm{M})$ \\
\hline Total no. $\neq$ & $27 / 99$ & 40 & 56 & 106 & 30 & 24 & 42 & 11 & 41 & 36 & 88 & 35 & 32 & \\
\hline Percentage & $21 \cdot 4 / 78 \cdot 6$ & $31 \cdot 7$ & $44 \cdot 4$ & $84 \cdot 1$ & $23 \cdot 8$ & 19 & $33 \cdot 3$ & $8 \cdot 7$ & $32 \cdot 5$ & $28 \cdot 6$ & $91 \cdot 7$ & $27 \cdot 8$ & $25 \cdot 4$ & \\
\hline
\end{tabular}

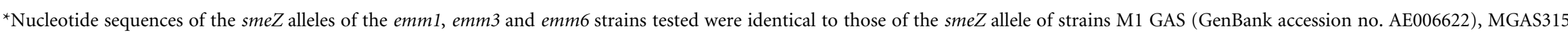
(AE014169) and MGAS10394 (NC_006086), respectively. A specific designation has not been attributed to these alleles.

$\dagger$ Also resistant to tetracycline.

$\ddagger$ Total number and frequency of each toxin gene in the entire collection of strains, except for smeZ, which was tested in 96 of 126 strains. 
case), were the most frequent clinical manifestations of invasive cases ( 11 of $27 ; 40 \cdot 7 \%$ ). Other diagnoses of invasive infections included arthritis (five cases), bacteraemia without specific focus (four cases), pneumonia (three cases), and one case each of meningitis, peritonitis, endocarditis and psoas abscess. STSS was recorded in four of the invasive cases (two cases of NF, one case of endocarditis and one case of bacteraemia without specific focus). The clinical presentations or sources of isolation of the 99 non-invasive isolates were skin-wound infections and impetigo $(n=37)$, genital infections $(n=20)$, otitis $(n=19)$, abscesses $(n=11$; 10 cutaneous and one tonsilar), pharyngitis $(n=10)$ and other sources including urine and eyes $(n=2)$.

The median age of patients with invasive infections was 55 years (mean 51.2 years; range 10 months to 89 years), whereas in the non-invasive group the median age was 8 years (mean 22.9 years; range 1 month to 91 years). The male to female ratio was $1 \cdot 5: 1$ and $1: 1$ for the invasive and non-invasive groups, respectively. At least one comorbid disease or local predisposing factor was found in $20(80 \%)$ out of 25 patients with available data in the invasive group. In one of the paediatric patients, invasive infection was associated with varicella, whereas, among adult patients, underlying conditions included skin trauma (eight cases), intravenous drug use and HIV infection (four cases, two with STSS and NF), cancer (four cases), peripheral vascular disease (three cases), immunosuppressive therapy (two cases), alcohol abuse (two cases) and diabetes mellitus (one case). Mortality occurred in three of $27(11 \%)$ patients with invasive infections, two of whom had STSS. Therefore, in agreement with previously published reports (Kao et al., 2005; O'Brien et al., 2002), most patients with invasive GAS infection were adults and a large proportion had underlying conditions, with HIV infection and injection drug use among the most frequent. Invasive GAS infection associated with injection drug use appears to be increasing in some countries (Efstratiou et al., 2003) and epidemics among this group have been documented (Léchot et al., 2001). It should be noted that, during the period studied, a local outbreak of invasive disease was detected among injecting drug users (Sierra et al., 2006). In this work, all invasive emm25.2 isolates were isolated from injecting drug users and were part of the local outbreak. Interestingly, an outbreak of invasive infection among drug users in Switzerland was reportedly caused by a specific emm 25 clone (Bohlen et al., 2000).

\section{Distribution of emm types}

In total, $29 \mathrm{emm}$ types were identified among the 126 isolates (Table 2), of which emm1 (22 isolates, $17 \cdot 5 \%$ ) was the most prevalent, followed by emm3 (11 isolates, $8 \cdot 7 \%$ ), emm4 (11 isolates, $8 \cdot 7 \%$ ), emm12 (nine isolates, $7 \cdot 1 \%$ ), emm 28 (nine isolates, $7 \cdot 1 \%$ ), emm11 (eight isolates, $6 \cdot 3 \%$ ) and emm77 (eight isolates, 6.3\%). These seven emm types accounted for $61.9 \%$ of isolates and have been reported to be among the most prevalent in USA, Canada and Europe (O'Brien et al., 2002; Schmitz et al., 2003; Tyrrell et al., 2002; Vlaminckx et al., 2003).
Most isolates presented emm sequences that were identical to those of the corresponding CDC emm reference strains. Seven types (emm3, emm18, emm25, emm29, emm43, emm49 and emm83) were exclusively represented by allelic variants of the reference strains. Different subtypes within a type were only found in types emm1, emm3, emm4 (two subtypes each) and emm12 (five subtypes). Three subtypes designated emm1.25, emm12.27 and emm83.4 were described for the first time in this study.

A total of 15 different emm types were identified in the 27 invasive isolates. All but two represented by single isolates were also present among non-invasive isolates. The most prevalent emm types were emm3 $(14 \cdot 8 \%)$, emm4 $(11 \cdot 1 \%)$, emm25 (11.1\%), emm77 (11.1\%), emm1 (7·4\%), emm6 $(7 \cdot 4 \%)$ and $\mathrm{emm} 9(7 \cdot 4 \%)$. A diversity of emm types was detected in isolates from the six patients with the most severe forms of infection (NF and SSTS), including two emm 25 and one each of emm1, emm4, emm11 and emm22. In the 99 non-invasive isolates, 27 different emm types were found, the most prevalent being emm1 $(20 \cdot 2 \%)$, emm4 $(8 \cdot 1 \%)$,

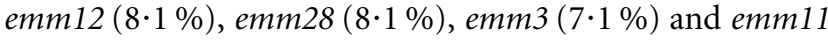
$(7 \cdot 1 \%)$.

No significant differences in emm type distribution were found between invasive and non-invasive isolates, as has been described recently (Descheemaeker et al., 2000; Haukness et al., 2002; Ho et al., 2003; Johnson et al., 2002; Kao et al., 2005). However, earlier reports identified a shift in dominant serotypes coincident with an increase in severe GAS infections, with M1 and M3 being those most frequently isolated from invasive cases (Johnson et al., 1992; Schwartz et al., 1990). The emergence of clones with increased virulence was proposed to explain the observed changes in GAS epidemiology (Musser et al., 1993, 1995).

Over the study period, there were no annual variations in the prevalence of the most common emm types. Certain subtypes such as emm11.0, emm25.2 and the newly identified emm1.25 were only found in years 2002 and 2003.

\section{Distribution of SAgs and toxin gene profiles}

Among the toxin genes that are thought to be chromosomally encoded, speB and speF were found in all isolates. As shown in Table 2, speG was found in $106(84 \cdot 1 \%)$ isolates and speJ was only found in $42(33.3 \%)$ isolates. smeZ was identified in $88(91.7 \%)$ of 96 isolates tested. DNA sequence analysis revealed the presence of 23 different $s m e Z$ alleles. Five $s m e Z$ alleles designated $s m e Z-35-s m e Z$-39 were identified for the first time in this study. As described by Proft et al. (2000), there was a strong association between the smeZ allele and the emm type. Five $s m e Z$ alleles (smeZ-3, smeZ-4, smeZ-13, smeZ-17 and smeZ-37) were each found in isolates of two different $\mathrm{emm}$ types. Isolates within an $\mathrm{emm}$ type carried the same smeZ allele except for the emm50 and emm 4 isolates (Table 2). The genes speA, speC, speH, speI, speL, speM and ssa, which have been found to be associated with prophage elements, were present at variable frequencies 
(Table 2). Significant differences were only found for ssa, which was more frequent in isolates from invasive cases $(P<0 \cdot 05$, Odds' ratio $2 \cdot 5 ; 95 \%$ confidence interval, $1 \cdot 27$ $4 \cdot 91)$. This correlation probably reflects the association of the ssa gene with the most prevalent emm types found in invasive infections (emm3, emm25 and emm4). Sequence analysis of the speA gene revealed four different alleles (Nelson et al., 1991), with a limited distribution of speA2, speA3 and speA4 in emm 1, emm 3 and emm6 isolates, respectively. The speA1 allele was identified in single isolates of three distinct emm types (emm18, emm4 and emm49).

The analysis revealed that isolates of the same emm type shared a common toxin gene profile (emm types $1,3,6,9$, $59,64,87$ and 89 ) or a predominant numerical profile (Table 2). Differences in toxin gene profiles between isolates of the same emm type involved the presence or absence of only one or two toxin genes compared with the dominant profile, except for the emm4.2 isolate, which differed in four toxin genes from emm4.0 isolates.

Although the number of isolates studied was limited, no significant difference in toxin gene profile was found between invasive and non-invasive isolates. Two recent European studies involving a larger number of isolates found that, despite the presence of several different toxin gene profiles within an emm type, each emm type was characterized by a predominance of one or two toxin gene profiles, reflecting the spread of few invasive clones throughout the world (Schmitz et al., 2003; Vlaminckx et al., 2003).

\section{Antimicrobial susceptibility}

All isolates were susceptible to penicillin $\mathrm{G}$ and cefotaxime. Erythromycin resistance was found in 35 isolates $(27 \cdot 8 \%)$, tetracycline resistance in 32 isolates $(25.4 \%)$ and resistance to both agents in 15 isolates $(11.9 \%)$. Four isolates $(3 \cdot 2 \%)$ showed reduced susceptibility to levofloxacin (MIC $\left.2-3 \mu \mathrm{g} \mathrm{ml}^{-1}\right)$.

Seventeen $(48.6 \%)$ of the erythromycin-resistant isolates expressed the $M$ phenotype and all of them harboured mef(A). The cMLSb phenotype was observed in $15(42 \cdot 8 \%)$ isolates, in association with $\mathrm{erm}(\mathrm{B})$ in all but one, which carried erm(TR). Three $(8.6 \%)$ isolates presented iMLSb; two of these carried erm(B) and the third carried erm(TR). The most prevalent emm types of erythromycin-resistant isolates were emm4, emm11, emm12 (four subtypes), emm 25 and emm75. Together, these five emm types represented $80 \%$ of the erythromycin-resistant isolates, but only $7 \cdot 7 \%$ of the susceptible isolates. All the emm4, emm 12 and emm75 erythromycin-resistant isolates harboured the mef(A) gene, whereas all emm11 and emm25 isolates carried the erm(B) gene. These data suggested that the high rate of erythromycin resistance was caused by the spread of a limited number of clones. An association between certain $\mathrm{emm}$ types and erythromycin resistance or macrolide resistance mechanisms has been documented (Albertí et al., 2003;
Zampaloni et al., 2003) and may possibly reflect the spread of resistant clones. In Spain, a strong association has been reported between emm types 4 and 75 and erythromycin resistance (Albertí et al., 2003; Pérez-Trallero et al., 1999).

A total of 32 tetracycline-resistant isolates were detected, belonging to 14 different $\mathrm{emm}$ types. The resistance determinant tet $(\mathrm{M})$ was present in 18 isolates $(56 \cdot 2 \%)$, tet $(\mathrm{O})$ in six isolates $(18 \cdot 7 \%)$, and one isolate carried both genes. None of the remaining seven tetracycline-resistant isolates carried either $\operatorname{tet}(\mathrm{M})$ or $\operatorname{tet}(\mathrm{O})$. tet $(\mathrm{O})$ was found exclusively in emm77.0 isolates.

Among the 15 isolates that were resistant to both erythromycin and tetracycline, $\operatorname{erm}(\mathrm{B})$ and $\operatorname{tet}(\mathrm{M})$ were found in six isolates, $\operatorname{erm}(\mathrm{TR})$ and $\operatorname{tet}(\mathrm{O})$ in one isolate and $\operatorname{erm}(\mathrm{TR})$ and tet $(\mathrm{M})$ in one isolate. Seven isolates, all emm11, carried $\mathrm{erm}$ (B), but none of the tetracycline resistance genes studied. None of the strains presenting the $\mathrm{M}$ phenotype were resistant to tetracycline.

The frequency of erythromycin-resistant isolates showed a rising trend over the study period $(16.6 \%$ in $1999,19 \%$ in $2000,21 \%$ in $2001,34 \cdot 6 \%$ in 2002 and $38.8 \%$ from January to June 2003). A high frequency of erythromycin resistance has been found in Spain (29.7\%) (Alós et al., 2003) and other European countries, including Italy $(35 \cdot 8 \%$; Dicuonzo et al., 2002) and France (22.4\%; Bingen et al., 2004), in contrast with the low rates observed in other countries such as the USA (6.8\%; Richter et al., 2005). The frequency of tetracycline resistance fluctuated year-to-year $(33.3 \%$ in $1999,19 \%$ in $2000,15 \cdot 8 \%$ in $2001,34 \cdot 6 \%$ in 2002 and $24 \cdot 2 \%$ from January to June 2003$)$. Multiclonal dissemination and high rates of tetracycline resistance have been reported from other countries (de Melo et al., 2003; Jasir et al., 2000).

The proportions of resistant isolates were similar in the invasive and non-invasive groups for both erythromycin (22.2\% invasive versus $29 \cdot 3 \%$ non-invasive) and tetracycline (33.3\% invasive versus $23 \cdot 2 \%$ non-invasive).

A number of reports during the last few years have described an increased prevalence of isolates with reduced susceptibility to fluoroquinolones (ciprofloxacin MIC $\geqslant 2 \mu \mathrm{g} \mathrm{ml}{ }^{-1}$ ) (Albertí et al., 2005; Malhotra-Kumar et al., 2005; Powis et al., 2005). In the present study, four isolates of three different emm types (emm3.1, emm6.0 and emm12.27) showed reduced susceptibility to levofloxacin (MIC 2-3 $\mu \mathrm{g}$ $\mathrm{ml}^{-1}$ ) and carried mutational changes in parC. A detailed characterization of these isolates has been published previously (Rivera et al., 2005).

In conclusion, the overall emm-type distribution found in this study was comparable with previous reports from North America and Europe. We found no differences in emm type distribution among isolates causing invasive or non-invasive infection. In addition, isolates of the same emm type presented restricted genetic variation with respect to $\mathrm{SAg}$ 
profiles. The erythromycin resistance rate presented a rising trend over the period studied and involved the spread of a limited number of clones. The SAg and antibiotic resistance genes thus appeared to be associated with the emm type and were independent of the disease type.

\section{ACKNOWLEDGEMENTS}

We would like to thank A. Lacal for clinical data collection and F. Grünbaum for statistical assistance.

\section{REFERENCES}

Åkesson, P., Rasmussen, M., Mascini, E. \& 8 other authors (2004). Low antibody levels against cell wall-attached proteins of Streptococcus pyogenes predispose for severe invasive disease. J Infect Dis 189, 797-804.

Albertí, S., García-Rey, C., Domínguez, M. A., Aguilar, L., Cercenado, E., Gobernado, M., Garcia-Perea, A. \& The Spanish Surveillance Group for Respiratory Pathogens (2003). Survey of emm gene sequences from pharyngeal Streptococcus pyogenes isolates collected in Spain and their relationship with erythromycin susceptibility. J Clin Microbiol 41, 2385-2390.

Albertí, S., Cortés, G., García-Rey, C., Rubio, C., Baquero, F., GarciaRodríguez, J. A., Bouza, E., Aguilar, L. \& The Spanish Surveillance Group for Respiratory Pathogens (2005). Streptococcus pyogenes pharyngeal isolates with reduced susceptibility to ciprofloxacin in Spain: mechanisms of resistance and clonal diversity. Antimicrob Agents Chemother 49, 418-420.

Alós, J. I., Aracil, B., Oteo, J., Gómez-Garcés, J. L. \& The Spanish Group for the Study of Infection in the Primary Health Care Setting (IAP-SEIMC) (2003). Significant increase in the prevalence of erythromycin-resistant, clindamycin- and miocamycin-susceptible (M phenotype) Streptococcus pyogenes in Spain. J Antimicrob Chemother 51, 333-337.

Banks, D. J., Beres, S. B. \& Musser, J. M. (2002). The fundamental contribution of phages to GAS evolution, genome diversification and strain emergence. Trends Microbiol 10, 515-521.

Basma, H., Norrby-Teglund, A., Guedez, Y., McGeer, A., Low, D. E., El-Ahmedy, O., Schwartz, B. \& Kotb, M. (1999). Risk factors in the pathogenesis of invasive group A streptococcal infections: role of protective humoral immunity. Infect Immun 67, 1871-1877.

Beall, B., Facklam, R. \& Thompson, T. (1996). Sequencing emmspecific PCR products for routine and accurate typing of group A streptococci. J Clin Microbiol 34, 953-958.

Bingen, E., Bidet, P., Mihaila-Amrouche, L., Doit, C., Forcet, S., Brahimi, N., Bouvet, A. \& Cohen, R. (2004). Emergence of macrolideresistant Streptococcus pyogenes strains in French children. Antimicrob Agents Chemother 48, 3559-3562.

Bohlen, L. M., Muhlemann, K., Dubuis, O., Aebi, C. \& Tauber, M. G. (2000). Outbreak among drug users caused by a clonal strain of group A streptococcus. Emerg Infect Dis 6, 175-179.

Chatellier, S., Ihendyane, N., Kansal, R. G., Khambaty, F., Basma, H., Norrby-Teglund, A., Low, D. E., McGeer, A. \& Kotb, M. (2000). Genetic relatedness and superantigen expression in group A Streptococcus serotype M1 isolates from patients with severe and nonsevere invasive diseases. Infect Immun 68, 3523-3534.

Cunningham, M. W. (2000). Pathogenesis of group A streptococcal infections. Clin Microbiol Rev 13, 470-511.

de Melo, M. C. N., Sá Figueiredo, A. M. \& Ferreira-Carvalho, B. T. (2003). Antimicrobial susceptibility patterns and genomic diversity in strains of Streptococcus pyogenes isolated in 1978-1997 in different Brazilian cities. J Med Microbiol 52, 251-258.

Descheemaeker, P., Van Loock, F., Hauchecorne, M., Vandamme, P. \& Goossens, H. (2000). Molecular characterisation of group A streptococci from invasive and non-invasive disease episodes in Belgium during 1993-1994. J Med Microbiol 49, 467-471.

Dicuonzo, G., Fiscarelli, E., Gherardi, G., Lorino, G., Battistoni, F., Landi, S., De Cesaris, M., Petitti, T. \& Beall, B. (2002). Erythromycinresistant pharyngeal isolates of Streptococcus pyogenes recovered in Italy. Antimicrob Agents Chemother 46, 3987-3990.

Efstratiou, A., Emery, M., Lamagni, T. L., Tanna, A., Warner, M. \& George, R. C. (2003). Increasing incidence of group A streptococcal infections amongst injecting drug users in England and Wales. J Med Microbiol 52, 525-526.

Facklam, R. F., Martin, D. R., Lovgren, M. \& 8 other authors (2002). Extension of the Lancefield classification for group A streptococci by addition of 22 new $M$ protein gene sequence types from clinical isolates: emm103 to emm124. Clin Infect Dis 34, 28-38.

Haukness, H. A., Tanz, R. R., Thomson, R. B., Jr \& 7 other authors (2002). The heterogeneity of endemic community pediatric group A streptococcal pharyngeal isolates and their relationship to invasive isolates. J Infect Dis 185, 915-920.

Ho, P. L., Johnson, D. R., Yue, A. W. Y., Tsang, D. N. C., Que, T. L., Beall, B. \& Kaplan, E. L. (2003). Epidemiologic analysis of invasive and noninvasive group A streptococcal isolates in Hong Kong. J Clin Microbiol 41, 937-942.

Hoge, C. W., Schwartz, B., Talkington, D. F., Breiman, R. F., MacNeill, E. M. \& Englender, S. J. (1993). The changing epidemiology of invasive group A streptococcal infections and the emergence of streptococcal toxic shock-like syndrome. A retrospective populationbased study. JAMA (J Am Med Assoc) 269, 384-389.

Igwe, E. I., Shewmaker, P. L., Facklam, R. R., Farley, M. M., van Beneden, C. \& Beall, B. (2003). Identification of superantigen genes $s p e M, s s a$, and sme $Z$ in invasive strains of $\beta$-hemolytic group $\mathrm{C}$ and $\mathrm{G}$ streptococci recovered from humans. FEMS Microbiol Lett 229, 259-264.

Jasir, A., Tanna, A., Noorani, A., Mirsalehian, A., Efstratiou, A. \& Schalen, C. (2000). High rate of tetracycline resistance in Streptococcus pyogenes in Iran: an epidemiological study. J Clin Microbiol 38, 2103-2107.

Johnson, D. R., Stevens, D. L. \& Kaplan, E. L. (1992). Epidemiologic analysis of group A streptococcal serotypes associated with severe systemic infections, rheumatic fever, or uncomplicated pharyngitis. J Infect Dis 166, 374-382.

Johnson, D. R., Wotton, J. T., Shet, A. \& Kaplan, E. L. (2002). A comparison of group A streptococci from invasive and uncomplicated infections: are virulent clones responsible for serious streptococcal infections? J Infect Dis 185, 1586-1595.

Kao, C.-H., Chen, P.-Y., Huang, F.-L. \& 10 other authors (2005). Clinical and genetic analysis of invasive and non-invasive group A streptococcal infections in central Taiwan. J Microbiol Immunol Infect 38, $105-111$.

Kotb, M., Norrby-Teglund, A., McGeer, A. \& 9 other authors (2002). An immunogenetic and molecular basis for differences in outcomes of invasive group A streptococcal infections. Nat Med 8, 1398-1404.

Lamagni, T. L., Efstratiou, A., Vuopio-Varkila, J., Jasir, A. \& Schalén, C. (2005). The epidemiology of severe Streptococcus pyogenes associated disease in Europe. Euro Surveill 10, 179-184.

Léchot, P., Schaad, H. J., Graf, S., Täuber, M. \& Mühlemann, K (2001). Group A streptococcus clones causing repeated epidemics and endemic disease in intravenous drug users. Scand J Infect Dis 33, $41-46$. 
Li, Z., Sakota, V., Jackson, D., Franklin, A. R. \& Beall, B. (2003). Array of M protein gene subtypes in 1064 recent invasive group A streptococcus isolates recovered from the active bacterial core surveillance. J Infect Dis 188, 1587-1592.

Malhotra-Kumar, S., Lammens, C., Chapelle, S., Mallentjer, C., Weyler, J. \& Goossens, H. (2005). Clonal spread of fluoroquinolone non-susceptible Streptococcus pyogenes. J Antimicrob Chemother 55, 320-325.

McCormick, J. K., Pragman, A. A., Stolpa, J. C., Leung, D. Y. M. \& Schlievert, P. M. (2001). Functional characterization of streptococcal pyrogenic exotoxin J, a novel superantigen. Infect Immun 69, 1381-1388.

Musser, J. M., Kapur, V., Kanjilal, S. \& 11 other authors (1993). Geographic and temporal distribution and molecular characterization of two highly pathogenic clones of Streptococcus pyogenes expressing allelic variants of pyrogenic exotoxin A (scarlet fever toxin). J Infect Dis 167, 337-346.

Musser, J. M., Kapur, V., Szeto, J., Pan, X., Swanson, D. S. \& Martin, D. R. (1995). Genetic diversity and relationships among Streptococcus pyogenes strains expressing serotype M1 protein: recent intercontinental spread of a subclone causing episodes of invasive disease. Infect Immun 63, 994-1003.

National Committee for Clinical Laboratory Standards (2003). Performance standards for antimicrobial disk susceptibility tests-eighth informational supplement, Approved standard M2-A8. Wayne, PA: NCCLS.

Nelson, K., Schlievert, P. M., Selander, R. K. \& Musser, J. M. (1991). Characterization and clonal distribution of four alleles of the speA gene encoding pyrogenic exotoxin A (scarlet fever toxin) in Streptococcus pyogenes. J Exp Med 174, 1271-1274.

Ng, L.-K., Martin, I., Alfa, M. \& Mulvey, M. (2001). Multiplex PCR for the detection of tetracycline resistant genes. Mol Cell Probes 15, 209-215.

Norrby-Teglund, A., Chatellier, S., Low, D. E., McGeer, A., Green, K. \& Kotb, M. (2000). Host variation in cytokine responses to superantigens determine the severity of invasive group A streptococcal infection. Eur J Immunol 30, 3247-3255.

Norrby-Teglund, A., Thulin, P., Gan, B. S., Kotb, M., McGeer, A., Andersson, J. \& Low, D. E. (2001). Evidence for superantigen involvement in severe group A streptococcal tissue infections. J Infect Dis 184, 853-860.

O'Brien, K. L., Beall, B., Barrett, N. L. \& 8 other authors (2002). Epidemiology of invasive group A streptococcus disease in the United States, 1995-1999. Clin Infect Dis 35, 268-276.

Pan, X.-S. \& Fisher, L. M. (1996). Cloning and characterization of the parC and parE genes of Streptococcus pneumoniae encoding DNA topoisomerase IV: role in fluoroquinolone resistance. J Bacteriol 178, 4060-4069.

Pérez-Trallero, E., Marimón, J. M., Montes, M., Orden, B. \& de Pablos, M. (1999). Clonal differences among erythromycin-resistant Streptococcus pyogenes in Spain. Emerg Infect Dis 5, 235-240.

Powis, J., McGeer, A., Duncan, C. \& 8 other authors (2005). Prevalence and characterization of invasive isolates of Streptococcus pyogenes with reduced susceptibility to fluoroquinolones. Antimicrob Agents Chemother 49, 2130-2132.

Proft, T., Moffatt, S. L., Weller, K. D., Paterson, A., Martin, D. \& Fraser, J. D. (2000). The streptococcal superantigen SMEZ exhibits wide allelic variation, mosaic structure, and significant antigenic variation. $J$ Exp Med 191, 1765-1776.

Proft, T., Arcus, V. L., Handley, V., Baker, E. N. \& Fraser, J. D. (2001). Immunological and biochemical characterization of streptococcal pyrogenic exotoxins I and J (SPE-I and SPE-J) from Streptococcus pyogenes. J Immunol 166, 6711-6719.

Proft, T., Webb, P. D., Handley, V. \& Fraser, J. D. (2003). Two novel superantigens found in both group A and group C Streptococcus. Infect Immun 71, 1361-1369.

Reinert, R. R., Lütticken, R., Bryskier, A. \& Al-Lahham, A. (2003). Macrolide-resistant Streptococcus pneumoniae and Streptococcus pyogenes in the pediatric population in Germany during 20002001. Antimicrob Agents Chemother 47, 489-493.

Richter, S. S., Heilmann, K. P., Beekmann, S. E., Miller, N. J., Miller, A. L., Rice, C. L., Doern, C. D., Reid, S. D. \& Doern, G. V. (2005). Macrolide-resistant Streptococcus pyogenes in the United States, 2002-2003. Clin Infect Dis 41, 599-608.

Rivera, A., Rebollo, M., Sánchez, F., Navarro, F., Miró, E., Mirelis, B. \& Coll, P. (2005). Characterisation of fluoroquinolone-resistant clinical isolates of Streptococcus pyogenes in Barcelona, Spain. Clin Microbiol Infect 11, 759-761.

Ruoff, K. L., Whiley, R. A. \& Beighton, D. (2003). Streptococcus. In Manual of Clinical Microbiology, 8th edn, pp. 405-421. Edited by P. R. Murray, E. J. Baron, J. H. Jorgensen, M. A. Pfaller \& R. H. Yolken. Washington, DC: American Society for Microbiology.

Schmitz, F.-J., Beyer, A., Charpentier, E., Normark, B. H., Schade, M., Fluit, A. C., Hafner, D. \& Novak, R. (2003). Toxin-gene profile heterogeneity among endemic invasive European group A streptococcal isolates. J Infect Dis 188, 1578-1586.

Schwartz, B., Facklam, R. R. \& Breiman, R. F. (1990). Changing epidemiology of group A streptococcal infection in the USA. Lancet 336, 1167-1171.

Seppälä, H., Nissinen, A., Yu, Q. \& Huovinen, P. (1993). Three different phenotypes of erythromycin-resistant Streptococcus pyogenes in Finland. J Antimicrob Chemother 32, 885-891.

Sierra, J. M., Sánchez, F., Castro, P. \& 16 other authors (2006). Group A streptococcal infections in injection drug users in Barcelona, Spain: epidemiologic, clinical, and microbiologic analysis of 3 clusters of cases from 2000 to 2003. Medicine (Baltimore) 85, 139-146.

Stevens, D. L. (2002). Streptococcal toxic shock syndrome. Clin Microbiol Infect 8, 133-136.

The Working Group on Severe Streptococcal Infections (1993). Defining the group A streptococcal toxic shock syndrome. Rationale and consensus definition. JAMA (J Am Med Assoc) 269, 390-391.

Tyrrell, G. J., Lovgren, M., Forwick, B., Hoe, N. P., Musser, J. M. \& Talbot, J. A. (2002). M types of group A streptococcal isolates submitted to the National Centre for Streptococcus (Canada) from 1993 to 1999. J Clin Microbiol 40, 4466-4471.

Vlaminckx, B. J. M., Mascini, E. M., Schellekens, J., Schouls, L. M., Paauw, A., Fluit, A. C., Novak, R., Verhoef, J. \& Schmitz, F. J. (2003). Site-specific manifestations of invasive group A streptococcal disease: type distribution and corresponding patterns of virulence determinants. J Clin Microbiol 41, 4941-4949.

Weber, P., Filipecki, J., Bingen, E., Fitoussi, F., Goldfarb, G., Chauvin, J. P., Reitz, C. \& Portier, H. (2001). Genetic and phenotypic characterization of macrolide resistance in group A streptococci isolated from adults with pharyngo-tonsillitis in France. J Antimicrob Chemother 48, 291-294.

Zampaloni, C., Cappelletti, P., Prenna, M., Vitali, L. A. \& Ripa, S. (2003). emm gene distribution among erythromycin-resistant and -susceptible Italian isolates of Streptococcus pyogenes. J Clin Microbiol 41, 1307-1310. 